\title{
Caracterización tecnológica y del talento humano de las empresas fabricantes de cerámica roja ubicadas en el área metropolitana de Cúcuta ${ }^{1}$
}

\author{
Jorge Sánchez Molina² $\mid$ John Freddy Gelves Diaz ${ }^{3} \mid$ Yebrail Alexis Romero Arcos ${ }^{4}$
}

Recibido:

Mayo 25 de 2012

Aceptado:

Septiembre 5 de 2012

${ }^{1}$ Los autores agradecen a todas las personas que colocaron su granito de arena para llevar a buen termino

el proyecto "Conformación e

implementación del cluster de la

cerámica de Norte de Santander",

al Departamento Administrativo de

Ciencia, Tecnología e innovación COLCIENCIAS por el apoyo brindado.

${ }^{2}$ Centro de Investigación de Materiales Cerámicos, Grupo de Investigación en Tecnología

Cerámica GITEC, Universidad

Francisco de Paula Santander,

Cúcuta-Colombia. Magister en Gerencia de empresas. Correo electrónico:

j_sanchezmolina@yahoo.es

${ }^{3}$ Centro de Investigación de Materiales Cerámicos, Grupo de Investigación en Tecnología

Cerámica GITEC, Universidad

Francisco de Paula Santander Cúcuta-Colombia. Ingeniero de Producción Industrial. Estudiante de Doctorado en Ciencia y Tecnología de Materiales. Correo electrónico: johngelves@yahoo.es

\footnotetext{
${ }^{4}$ Universidad Francisco de Paula

Santander, Cúcuta-Colombia. Ingeniero de Producción Industrial. Especialista en gestión de proyectos. Estudiante de Maestría en Gerencia de Empresas.Correo electrónico: alexis.romero@gmail.com
}

\section{Resumen}

El presente trabajo muestra como están conformadas las empresas fabricantes de cerámica roja de esta región de Colombia, desde el punto de vista productivo, tecnológico, económico y de talento humano que allí labora. La información consolidada aquí se ha basado en un muestreo representativo de las unidades productivas que se encuentran legalmente constituidas y registradas en la Cámara de comercio de Cúcuta. El panorama encontrado ha dejado ver que esta industria ha alcanzado su mayor periodo de crecimiento en la última década (2000. 2010), predominando la empresa de clase pequeña, con un especial énfasis en la fabricación de cerámicas no esmaltadas; se evidencia un nivel tecnológico mediobajo al ser comparado con uno de los principales referentes internacionales; algo similar se aprecia en el nivel de formación académica del personal con altos niveles de analfabetismo y una limitada participación de personal especializado.

Palabras clave: Cerámica roja, área metropolitana de Cúcuta, Cluster, vitrificado, horno colmena

\section{Abstract}

The objective of this article is to present the productive, technological, economic and human talent areas of the manufacturing companies of red clay in Norte de Santander-Colombia. This is attained by sampling the companies legally constituted and registered in the commerce chamber of Cúcuta. The results found show a growth in the ceramic industry in the last decade (2000 -2010), where the small companies prevail with special emphasis on the manufacture of unglazed ceramics; the technological level is medium-low compared with international standards, something similar happens with the education of the personnel, there's a high level of illiteracy and the specialized personnel is limited.

Keyword: Red cerámic, metropolitan area of Cúcuta, cluster, vitrified, kiln hive 


\section{Introducción}

$\mathrm{E}$ departamento Norte de Santander se caracteriza por poseer un gran potencial minero, el carbón, las arcillas, las calizas, feldespatos y fosfatos son algunos de los materiales que actualmente se extraen y se transforman en la región [1].

La ubicación geográfica del departamento, en la que se destaca la posición fronteriza, la abundancia y calidad de las arcillas han conllevado a que en la región se haya instalado una gran cantidad de unidades productivas dedicadas a la fabricación de materiales cerámicos, principalmente aquellos destinados a la industria de la construcción. Esta concentración geográfica de industrias cerámicas, hacen de este sector uno de los más estratégicos y promisorios para el departamento, como muestra de esto, se tiene que el departamento, ocupa el primer lugar en exportaciones en la mayoría de subpartidas arancelarias que hacen parte del capitulo 69, de acuerdo con las estadísticas publicadas por Proexport en los últimos años [2-5].

La transformación que ha tenido el sector cerámico regional en los últimos años ha conllevado a que no se tenga un conocimiento muy claro de los aspectos productivos, económicos, tecnológicos y sociales que se mueven en torno a la empresas que conforman este sector. Aunque se ha realizado varios estudios [6-11], estos solo se han enfocado a temas muy específicos a algunas pocas unidades productivas, lo cual no es garantía para hacer inferencias sobre todo el sector, debido a aspectos como versatilidad de técnicas de fabricación, tamaño de la fábrica, tipo de productos elaborados, ubicación geográfica dentro del área metropolitana, las cuales tienen gran influencia acorde a la literatura. La ejecución del proyecto denominado "Conformación del Cluster de la cerámica de Norte de Santander" el cual es la base del desarrollo del presente trabajo, permitió una caracterización más a fondo del sector cerámico regional, en temas como clasificación de empresas de acuerdo a la normatividad, clasificación de productos fabricados, procesos productivos utilizados, tecnologías empleadas, aspectos económicos y de talento humano que labora en esta industria [12-13].

\section{Materiales y métodos}

La investigación se desarrolló en la Zona Metropolitana de San José de Cúcuta conformada por los municipios de Cúcuta, El Zulia, Los Patios, Villa del Rosario y San Cayetano; siendo estos municipios los de mayor concentración de unidades productivas dedicadas a la fabricación de cerámica roja. La investigación realizada fue del tipo descriptivo con carácter exploratorio. La población tomada como objeto de estudio fueron las empresas fabricantes de cerámica roja ubicadas en esta región y registradas en la Cámara de Comercio de Cúcuta. Se realizó un muestreo de tipo probabilístico, estableciendo la muestra de acuerdo a la fórmula

$$
n=\frac{k^{2} * N^{*} p^{*} q}{k^{2^{*}} p^{*} q+N^{*} \varepsilon^{2}}
$$

Siendo

$$
\begin{aligned}
& \mathrm{p}=0.5 \text { (probabilidad de éxito) } \\
& \mathrm{q}=1-\mathrm{p}=0.5 \text { (probabilidad de fracaso) }
\end{aligned}
$$

De acuerdo a lo expuesto por Weiers [14], cuando no se tiene una proporción de $\mathrm{p}$, se debe ser moderado y tomar los valores de $\mathrm{p}=0.5$.

$$
\begin{gathered}
\alpha=95 \% \\
\mathrm{k}=\text { múltiplo de error estándar. Se selecciona } \\
\text { un nivel de confianza del } 95 \%, \mathrm{k}=1.96
\end{gathered}
$$


$\mathrm{e}=$ error o máxima diferencia entre la proporción muestral y la proporción de la población que se esta dispuesto a aceptar en el nivel de confianza, se asume un e $=10 \%$, que es utilizado para los estudios sociales.

$$
\begin{aligned}
& \mathrm{N}=\text { población, objeto de estudio } \\
& \mathrm{n}=\text { empresas de muestreo }
\end{aligned}
$$

De donde

$\mathrm{N}$ = población, objeto de estudio (59 empresas fabricantes de productos cerámicos)

$$
n=\frac{(1,96)^{2} * 59 * 0,5 * 0,5}{(1,96)^{2 *} * 0,5 * 0,5+\left(59 *(0,1)^{2}\right)}=37
$$

$\mathrm{n}=37$ empresas de fabricantes

La recolección de información se llevó a cabo, realizando visitas de campo a las empresas fabricantes de cerámica roja, utilizando las técnicas de observación directa, encuestas y entrevistas en algunos casos. El trabajo de captura de la información fue realizada por profesionales con experiencia en el tema, recopilando información de informantes claves tales como: gerentes de planta y de asociaciones (Induarcilla), jefes de producción y de operarios en cada una de las empresas seleccionadas, utilizando herramientas para recolección de evidencia fotográfica y formatos de registros de novedades de las observaciones encontradas. La información fue analizada por personal experto empleando principios de estadística descriptiva tales como medidas de tendencia central y graficación de resultados, con el apoyo de la aplicación Excel de Microsoft Corporation.

\section{Resultados y discusión}

Inicialmente se muestra las características generales de las empresas del sector, seguidamente de los aspectos de producción y tecnología, y, finalmente el tema relacionado con el talento humano.
La ley 905 de 2004 permite clasificar las empresas colombianas de acuerdo a criterios como nivel de activos y numero de empleados, en las figuras 1 y 2 se muestra los resultados obtenidos para las empresas que hacen parte del cluster de la cerámica del área metropolitana de Cúcuta (fabricantes de cerámica roja); las figuras 3 y 4 muestran aspecto relacionados con la existencia de la empresas y principales tipos de sociedades conformadas.

Figura 1. Rango de activos totales que manejan las empresas fabricantes de cerámica roja en unidades de salarios mínimos mensuales vigentes

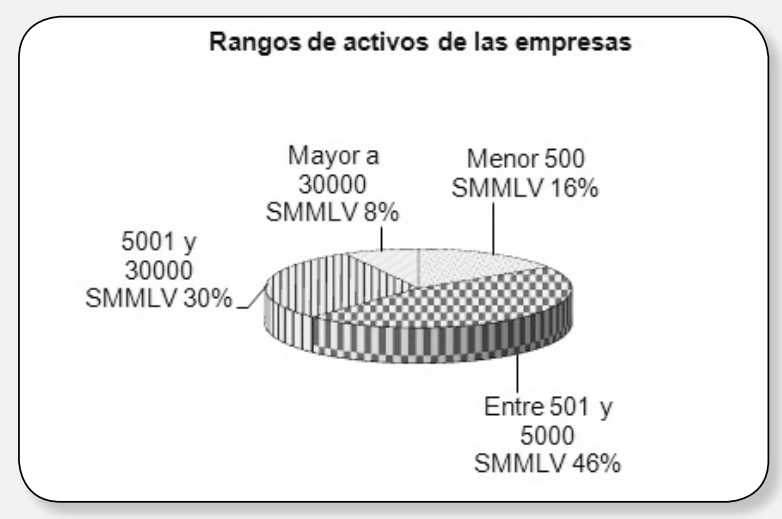

Figura 2. Número de empleados de las empresas fabricantes de cerámica roja del área metropolitana de Cúcuta

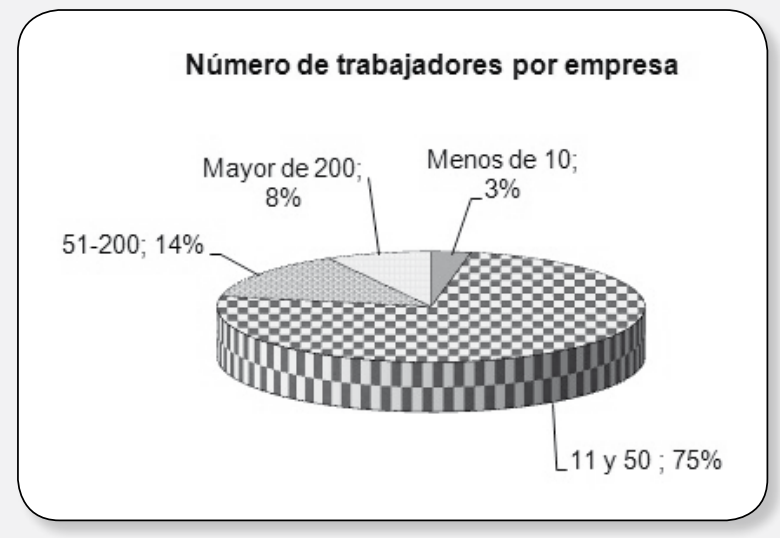




un $16 \%$ se clasifica como microempresa, el $30 \%$ como mediana empresa y un $8 \%$ como gran empresa. El número de empleados de las empresas del sector según la figura 2 se encuentra en su mayoría entre 11 y 50 empleados, que corresponde a la pequeña empresa, con $75 \%$; entre 51 y 200 con un $14 \%$ que corresponde a la mediana empresa. Se puede clasificar las empresas según su orden de importancia como: pequeña, mediana, microempresa y gran empresa.

Figura 3. Tiempo de funcionamiento de las empresas fabricantes de cerámica roja

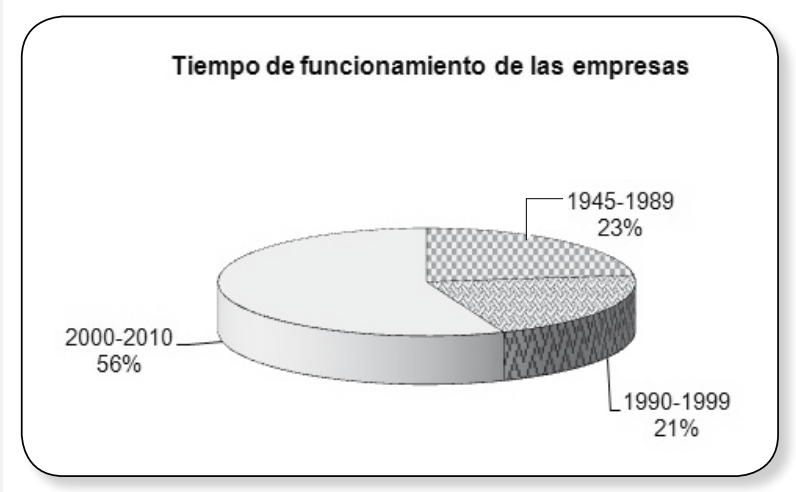

Figura 4. Tipo de sociedades de las empresas fabricantes de cerámica roja

Tipo de sociedad económica

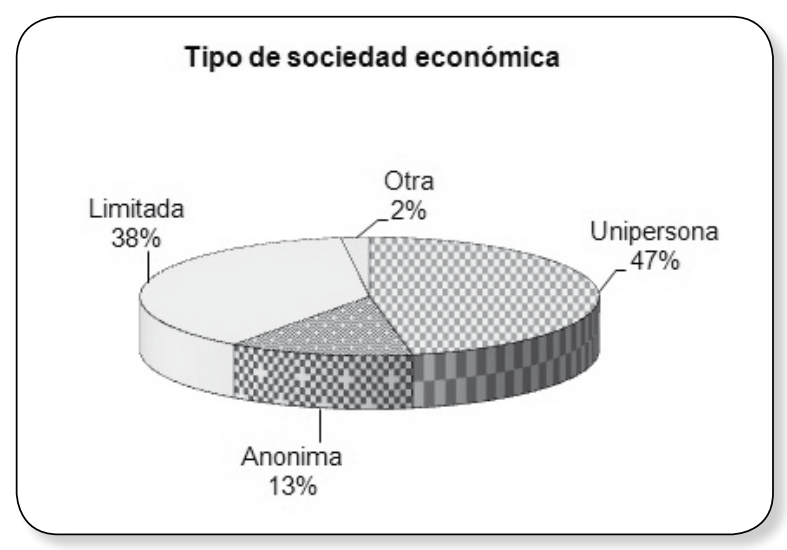

La figuras 3 y 4 dejan ver que gran parte de las empresas se han establecido en la última década $56 \%$, y que un gran porcentaje, $47 \%$ de las mismas son de un único dueño.

Como aspecto de importancia para caracterizar a las empresas fabricantes de cerámica roja, se determino el nivel de ingresos de las organizaciones que hacen parte de este conglomerado industrial. En la figura 5 se pueden apreciar los resultados.
Figura 5. Nivel de ingreso mensual de las empresas fabricantes de cerámica roja (Pesos)

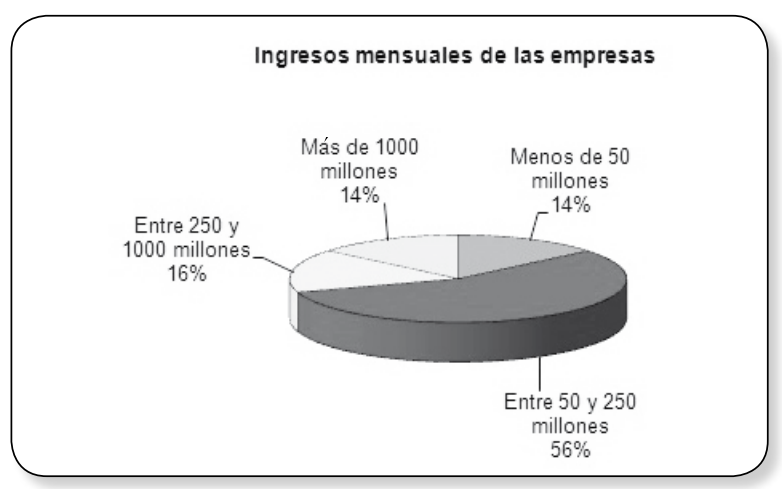

En la figura 5 se observa que el 30\% de las empresas reportan ingresos superiores a 250 millones de pesos mensuales, el 56\% está entre 50 y 250 millones de pesos mensuales; este valor de ingresos viene a corresponder a los ingresos de las empresas de tamaño pequeño [15].

Los niveles de capacidad instalada guardan una relación directa con los niveles de ingresos de las empresas del sector, la figura 6 muestra los rangos de capacidad instalada de las empresas fabricantes de cerámica roja de la región.

Figura 6. Capacidad instalada de las empresas en toneladas/mes

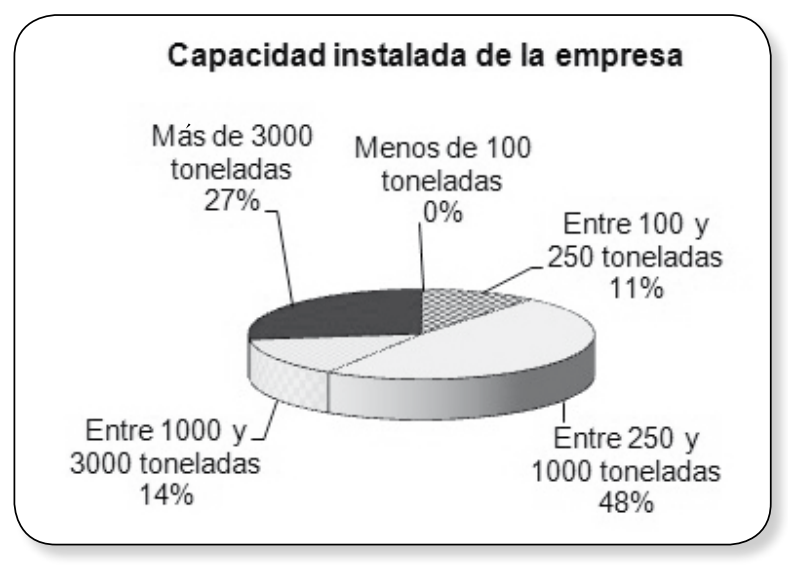

El $48 \%$ de las empresas tiene una capacidad instalada entre 250 y 1.000 toneladas/mes, el 27\% tiene una capacidad instalada superior a 3.000 toneladas $/ \mathrm{mes}$, lo que aproximadamente coincide con el porcentaje observado en la figura 5, con empresas que tienen ingresos superiores a 250 
millones de pesos. Al comparar la industria local con algunos referentes españoles como el grupo Uralita, Productos cerámicos Borja, Cerámicas Collado y del Ter cuyos niveles de consumo mensual están por encima de las 9600 toneladas mensuales; se puede apreciar que la capacidad instalada en la región es baja, de igual manera los ingresos mensuales son mucho mas bajos, siendo en promedio para las empresas españolas asociadas en Hispalyt de 800 millones de pesos, tomando como periodo de comparación el año 2009, [15-16].

El cluster de la cerámica del área metropolitana de Cúcuta se caracteriza por que la gran mayoría de productos fabricados están destinados a la industria de la construcción [17]. En la figura 7 se puede apreciar los productos (cerámica roja) que son fabricados por las empresas del sector.

Figura 7. Tipo de productos fabricados en las empresas

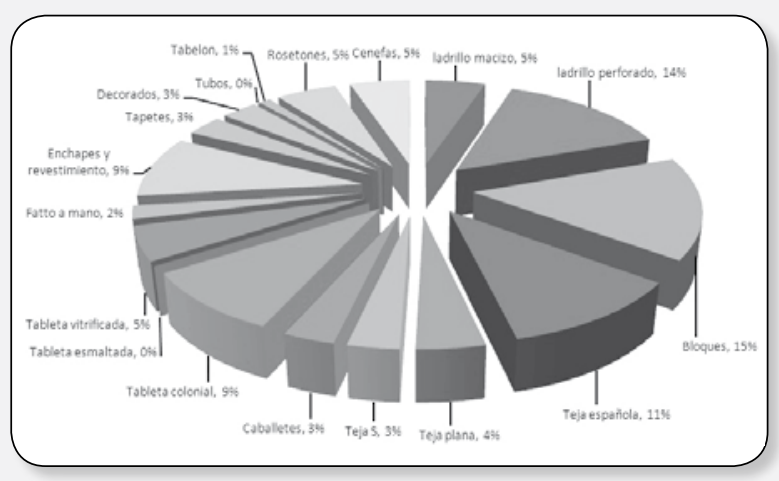

En la figura 7, se aprecia, que la mayor proporción de productos fabricados en las empresas corresponde a bloque (15\%), ladrillo perforado (14\%), Teja española (11\%), tableta colonial $(9 \%)$ y enchapes y revestimientos (9\%) y en menor proporción se encuentra los tubos $(0 \%)$, tabelon $(1 \%)$, tableta esmaltada $(0 \%)$. Se aclara que el $0 \%$ no significa que no se fabrique en las empresas, sino que en proporción, comparado con la producción de los otros productos, es insignificante. Los resultados mostrados son los que se recolectaron para inicios del año 2011, en épocas anteriores (años 2007 y 2008) las tejas y las tabletas vitrificadas eran las de mayor representatividad, pero debido al cierre del mercado venezolano por los problemas de tipo político hicieron que estos datos cambiaran significativamente [18].

En referencia al nivel de producción de los artículos elaborados por las empresas se encontró bastante divergencia entre los datos reportados por los empresarios, por tanto se realizó una clasificación de acuerdo a toneladas producidas mensualmente, metros cuadrados y unidades elaboradas, las figuras 8,9 y 10 muestran los resultados de esta clasificación

Figura 8. Participación de los productos sobre la producción promedio mensual de las empresas en Tonelada/mes

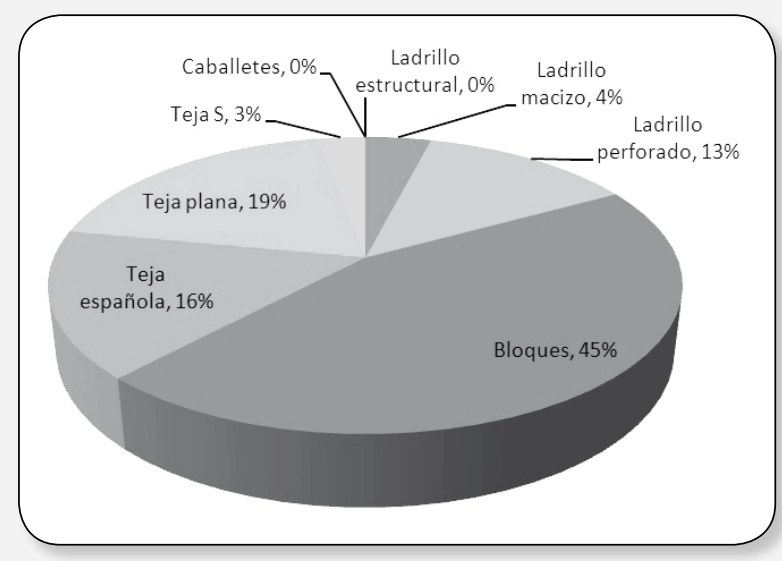

En las fábricas la mayor producción en toneladas/mes se reporta en la producción de bloques, con una proporción apreciable de $45 \%$, se observa en menor proporción la producción de ladrillo perforado, teja plana y la teja española. El resto de productos también se fabrican pero su proporción es mínima comparada con la producción de Bloques. Las condiciones favorables del mercado venezolano entre 2004 y 2008 tenían un panorama distinto al plasmado en la figura 8 , la creación de empresas en la época acorde a lo presentado en la figura 3 se enfocó a la fabricación de teja, puesto que este producto requería menores consumo de materia prima y costo de fletes por unidad, lo cual impulso significativamente la manufactura de este producto para su exportación, permitiéndole así ocupar el primer puesto en producción a nivel de piezas medidas en unidades, incluso a nivel general, acorde a las estadísticas de Proexport y diferentes trabajos realizados [3-6]. 
Las condiciones actuales no favorecen las exportaciones para estas empresas, por tanto han tenido que adaptarse a la demanda local, regional y nacional, la cual en la actualidad requiere principalmente material de cerramiento como el bloque, lo cual se refleja en los resultados aquí presentados; este panorama, no favorece los ingresos de las empresas puesto que para la fabricación de una teja española solo se requieren 1,25 Kilogramos de arcilla en promedio, mientras que para un bloque (Número 5) tradicional en promedio se requieren 4,50 kilogramos, esto sumado a los costos de transformación y al bajo valor agregado, ha conllevado a que muchas unidades productivas estuviesen en crisis financiera para las fechas donde se llevó a cabo la recolección de información. En la figura 9 se puede apreciar que la mayor proporción de producción en metros cuadrados es en tableta vitrificada con un $54 \%$. Un $35 \%$ es tableta colonial y un $11 \%$ de enchapes vitrificados. Es insignificante la producción de fatto a mano, comparado con la producción de tableta vitrificada.

Figura 9. Participación de los productos sobre la producción promedio mensual de las empresas en $\mathrm{m}^{2}$

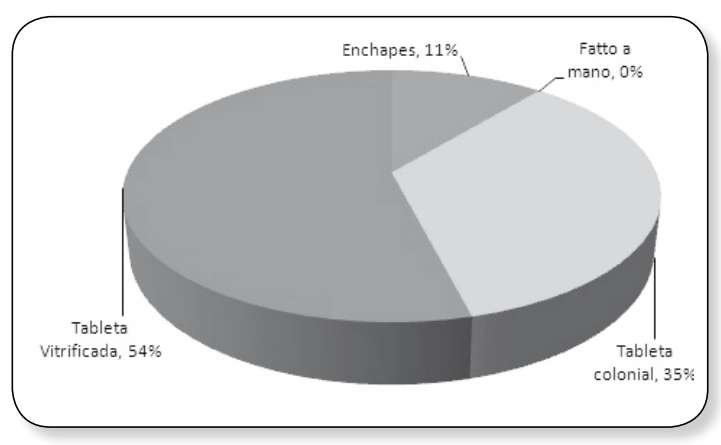

Figura 10. Participación de los productos sobre la producción promedio mensual de las empresas en unidades

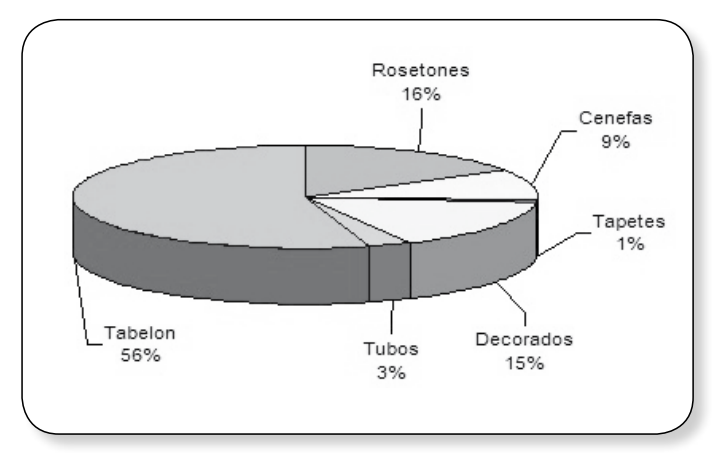

De los productos que se producen por unidades, el de mayor proporción es el tabelón con un $56 \%$, el resto de productos tiene una producción baja comparada con este producto.

Los clientes de las empresas fabricantes de cerámica roja son los que se muestran en la figura 11, de igual forma en la figura 12 se presentan los resultados de representatividad de los productos que son exportados.

Figura 11. Clientes de las empresas fabricantes de cerámica roja

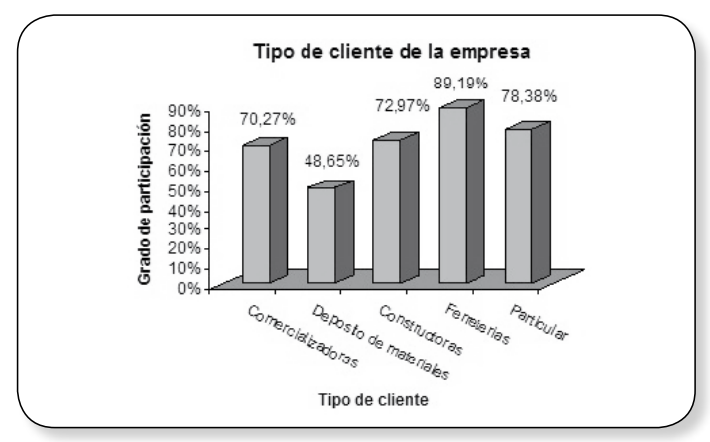

Figura 12. Representatividad de los productos exportados

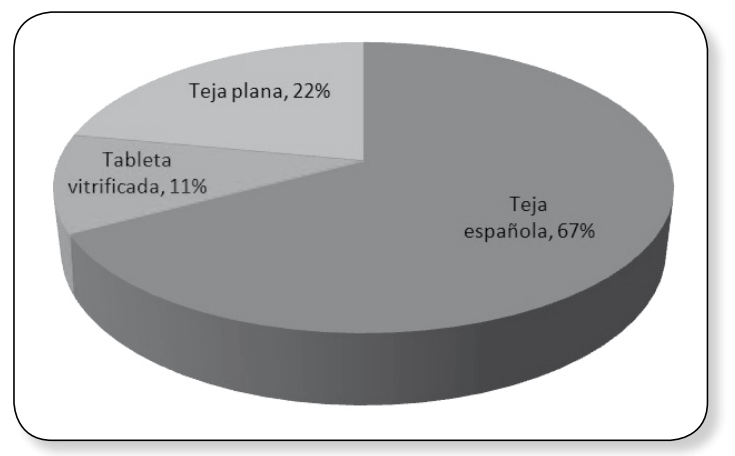

De los 15 productos (cerámica roja) que se producen en la región, solo tres se exportan. La mayoría es la teja española con un $67 \%$, seguido de teja plana con un $22 \%$ y tableta vitrificada $11 \%$. Se encontró que Venezuela es el principal mercado para la empresas del sector, en menor proporción se encuentra las exportaciones a Ecuador, Panamá, Estados Unidos y las islas del Caribe.

Los resultados de la caracterización de la tecnología empleada en las empresas fabricantes de cerámica roja del área metropolitana de Cúcuta se presenta a continuación, la figura 13 muestra los métodos 
Caracterización tecnológica y del talento humano de las empresas fabricantes de cerámica roja

de conformado empleados para la elaboración de las piezas. La figura 14 presenta el método de secado empleado y la figura 15 la tecnología empleada para la sinterización (la sinterización es uno de los métodos de fabricación más antiguos de metales y materiales cerámicos) de los artículos cerámicos.

Figura 13. Método empleado para el conformado de las piezas cerámicas

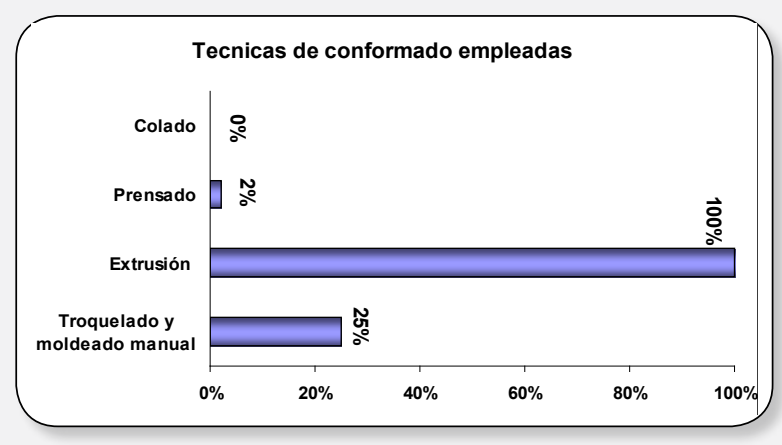

Figura 14. Métodos utilizados para el secado de los productos

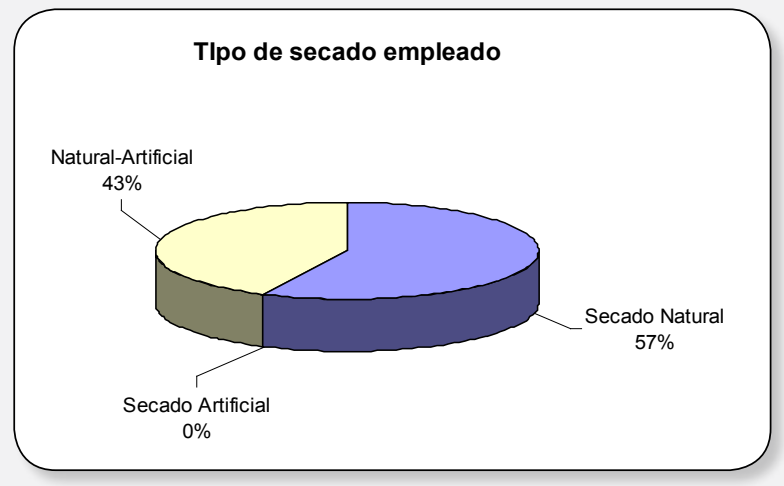

Figura 15. Hornos empleados en la empresa fabricantes de cerámica roja

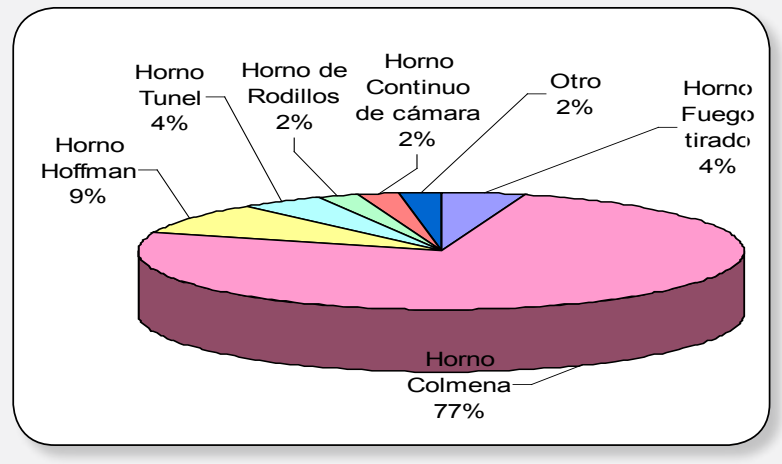

Según la figura 15 el tipo de horno más utilizado en las empresas fabricantes de cerámica roja de la región es el horno colmena o de llama invertida (77\%), en mínima proporción se pueden encontrar en las empresas hornos Hoffman (9\%), Horno túnel (4\%), Horno de fuego tirado (4\%), Horno de Rodillos (2\%), Hornos de cámara (2\%) y otro (2\%). Si se hace una comparación con España (empresas fabricantes de cerámica roja) se tiene que, los hornos túneles son el $86,72 \%$, Hoffman $11,95 \%$, intermitentes $0.87 \%$ y rodillos 0,46\% [19]. Haciendo el análisis se evidencia que en España predomina los hornos túneles con $86,72 \%$, mientras que en Cúcuta y su área metropolitana el colmena con $77 \%$ es el de mayor uso, y solamente el $4 \%$ son hornos túneles.

Los resultados de caracterización del talento humano se presentan en la figuras 16 a 18, las variables tenidas en cuenta fueron, representatividad de genero, nivel académico de los empleados del sector y densidad poblacional de las áreas de trabajo.

Figura 16. Representatividad del género en las empresas fabricantes de cerámica roja

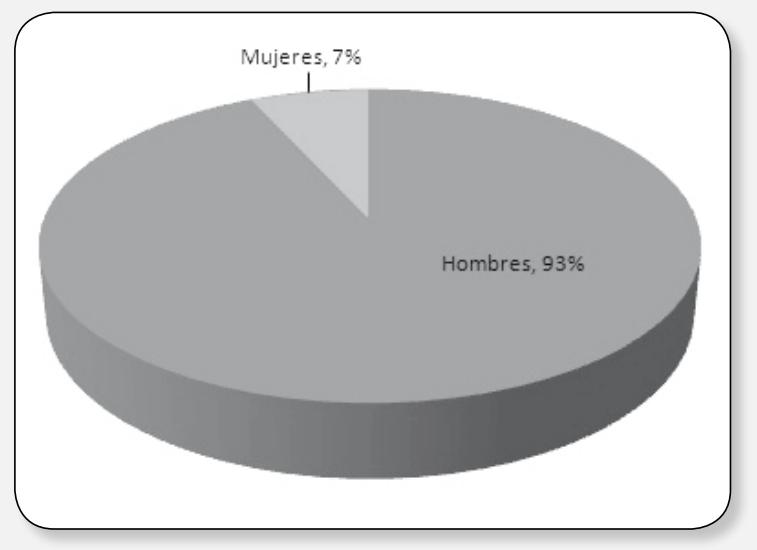

Figura 17. Nivel académico de los empleados que hacen parte de las empresas fabricantes de cerámica roja

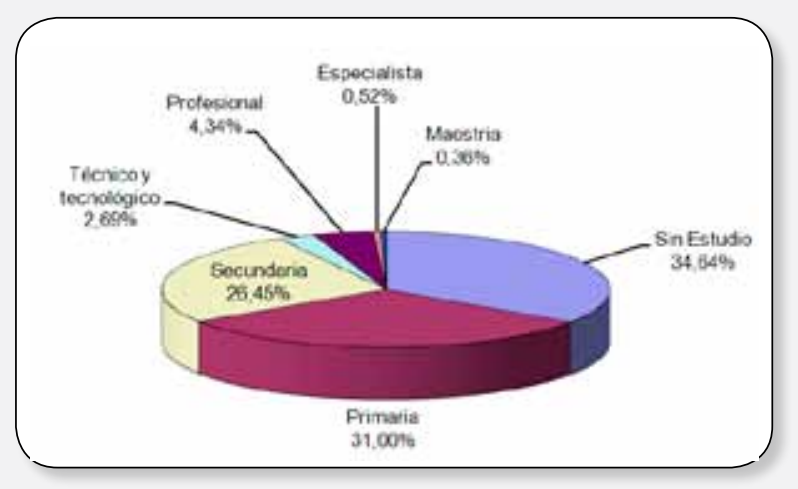


Figura 18. Densidad poblacional por secciones de trabajo

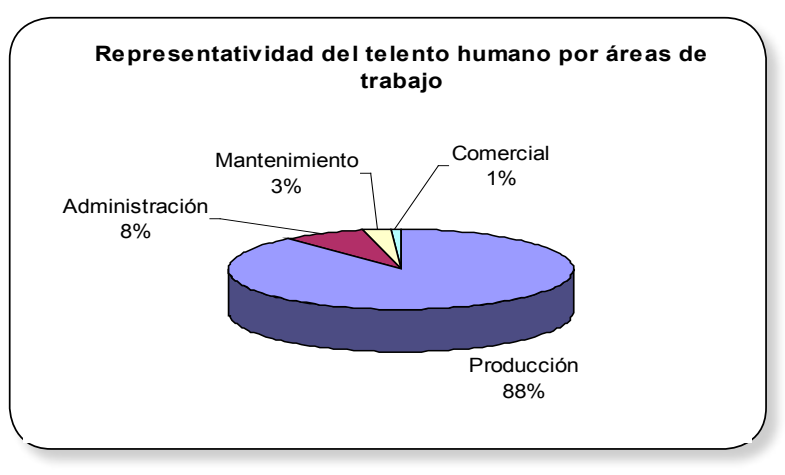

De las observación directa de los expertos se puede concluir que la mayor parte de la población que labora en estas empresas realiza actividades de gran esfuerzo físico (El 88\% de los empleados están en el área de producción), las tareas son arduas y agotadoras, debido a las altas temperaturas a las que se ven expuestos los operarios, principalmente las cuadrillas de cargue-descargue de hornos y los encargados de la dosificación de carbón a las hornillas, también son afectados por la presencia de polvo y humos del proceso de combustión en los hornos.

De las figuras 16 a 18 se observa con claridad el predominio del género masculino con un $93 \%$ de la población, las mujeres realizan labores de apoyo administrativo en la mayoría de los casos, en los centros de producción realizan tareas de fabricación de productos decorativos y artesanales, esta circunstancia se da por la necesidad de grandes esfuerzo físicos en las actividades laborales más no por algún tipo de discriminación de género. Se evidencia que más de la mitad de la población que labora en el sector $(65,6 \%)$ no sobrepasa el nivel de estudio de primaria, de igual modo el nivel de formación universitario que participa en el sector no supera el 8,0\%, del cual la participación de personal a nivel de posgrado no alcanza a superar el 1,0\%, dejando en evidencia la falta de capacidades para el desarrollo de nuevos productos y procesos de innovación que impacten en la competitividad del sector.

En materia de contratación laboral, se encontró que predomina la contratación a término fijo, la contratación directa se da en la mayoría de los casos, seguida de la contracción por cooperativas, en menor grado se encuentra la contratación por obra realizada; estas dos últimas formas se dan principalmente en el área de producción para las empresas más pequeñas, en especial en actividades de cargue y descargue del material.

Otros aspectos que resultan de gran importancia y que permiten tener una mayor caracterización del sector fueron obtenidos del presidente de la Asociación de Industriales de la Arcilla de Norte de Santander, el cual expreso que "Las empresas en su conjunto generan aproximadamente 7.500 empleos directos, consumen de 17.000 a 25.000 toneladas mensuales de carbón, demandan servicios de transporte a diferentes destinos del mercado local, nacional y de exportación de aproximadamente 80.000 toneladas al mes, el sector genera a la economía regional unos ingresos cercanos a los $\$ 25.600$ miles de millones de pesos. El valor total de la mano de obra generado en las industrias del sector es de $\$ 3.315$ miles de millones de pesos por mes, dando aportes de seguridad social, parafiscales, fondos de cesantías y pensión, pueden alcanzar el \$1.755 miles de millones de pesos por mes aproximadamente. Sin mencionar el aporte de impuestos de industria y comercio, contribuciones para alumbrado público, aportes consumo de energía e impuesto al valor agregado IVA, impuesto al patrimonio y en general el sector es un importante generador de recursos que imprime a la región una importante dinámica económica y social.

\section{Conclusiones}

La mayor parte de las empresas fabricantes de cerámica roja de la zona metropolitana de Cúcuta son pequeñas, de acuerdo a lo establecido en la ley 905 de 2004, le siguen en orden de importancia las medianas, micro y gran empresa. La producción de estas organizaciones esta destinada casi en su totalidad a la industria de la construcción, se estableció que gran parte de las empresas se conformaron en la última década, y una proporción significativa de estas, tienen 
un único dueño. La mayor parte de estas organizaciones $(70 \%)$ reciben ingresos mensuales inferiores a los 250 millones de pesos siendo mucho menor en comparación con las empresas españolas de tamaño pequeño, las principales razones se deben a mayores ventas y valor agregado de los productos españoles.

Los productos más elaborados por las empresas fabricantes de cerámica roja son: ladrillo perforado, bloque número 5 , teja curva y plana, tableta vitrificada, tableta colonial y fachaletas; de los cuales la teja curva es la de mayor volumen de exportación, seguido de la teja plana y la tableta vitrificada. El método más empleado para el conformado de los productos cerámicos es la extrusión, el secado de las piezas en su mayor parte se lleva a cabo de manera natural, los hornos más comunes son los de llama invertida, conocidos como colmena con un $77 \%$ de participación. Los sistemas automáticos son muy limitados, se puede afirmar que el nivel tecnológico de estas empresas es bajo en comparación con países como España e Italia, motivo por el cual está demostrado que los avances de las regiones más desarrolladas, están basadas en las capacidades que posea el recurso humano que lo conforma; estas tienen su pilar en el grado de formación académica que se ha adquirido. Por tanto, resulta de gran importancia para el sector cerámico del área metropolitana de Cúcuta; que las directivas de las diferentes organizaciones que conforman el gremio, las entidades académicas y gubernamentales regionales generen sinergia para la toma de medidas que conlleven a mejorar el nivel de formación académico del talento humano que labora en este sector tan importante para la economía de esta zona del país.

\section{Referencias Bibliográficas}

[1]. JESKAR LTDA. Inventario geológico minero, ambiental, tecnológico y empresarial de los minerales no energéticos del Norte de Santander, San José de Cúcuta, junio de 2000.
[2]. DEPARTAMENTO NACIONAL DE PLANEACIÓN. Agenda interna para la productividad y competitividad de Norte de Santander. [En línea]. Tomado el 20 de Julio de 2011. Disponible en http://www.mincomercio.gov.co/ eContent/documentos/Competitividad/ InsumosNortedeSantander.pdf

[3]. PROEXPORT COLOMBIA. Sistema de Información comercial. En línea, disponible en <http://www. colombiatrade.com.co/herramientas $>$

[4]. GRUPO DE INVESTIGACIÓN EN TECNOLOGÍA CERÁMICA. Conformación e implementación del cluster de la cerámica de Norte de Santander. San José de Cúcuta, año 2008. Universidad Francisco de Paula Santander, Facultad de ciencias básicas.

[5]. CALDERON ARTEAGA, Christian Hugo. Producción y comercialización del ladrillo [en línea]. [Tomado el 20 de noviembre de 2009]. Disponible en Internet <http:// www.monografias. com/trabajos 14/ladrillocolomb/ ladrillocolomb.shtml>

[6]. CARRILLO CÁRDENAS, Mónica y AREVALO MONROY, Iraní. Actualización de la información y puesta en marcha de una aplicación sistematizada para el sector cerámico del área metropolitana de san José de Cúcuta. Plan de estudios de Ingeniería de Producción Industrial. San José de Cúcuta. Universidad Francisco de Paula Santander. Facultad de Ingeniería. Año 2008.

SERVICIO NACIONAL DE APRENDIZAJE, SENA. Centro Nacional Minero. Regional Boyacá. Caracterización Ocupacional Industria de la Arcilla. Julio de 2003.

[8]. SEMILLERO DE INVESTIGACIÓN DE MATERIALES CERÁMICOS SIMAC. Diagnóstico de la Situación Actual de los Chircales Artesanales 
del Área Metropolitana de Cúcuta. Universidad Francisco de Paula Santander, Facultad de Ciencias Básicas, Grupo de Investigación en Tecnología Cerámica GITEC, San José de Cúcuta, año 2004.

[9]. BETANCUR Juan Pablo; GELVES DIAZ John Freddy. Diagnostico de los procesos de secado, cocción y manejo ambiental en las empresas productoras de tableta vidriada de la zona metropolitana de San José de Cúcuta. Trabajo de grado Ingeniería de producción industrial. Cúcuta. Universidad Francisco de Paula Santander, Facultad de ingenierías. Año 2006.

[10]. PRATO CRUZ Elcy Joanna "Planteamiento de modelos productivos para la estandarización de los procesos de producción de las Mipymes del sector cerámico del área metropolitana de San José de Cúcuta". Grupo de Investigación en Tecnología Cerámica GITEC. Universidad Francisco de Paula Santander, Facultad de Ciencias Básicas, Año 2008.

[11]. FORNES RIVERA Manuel Y CANCINO RINCÓN Leonardo "Determinación del estado actual de la administración de talento humano en el sector cerámico de la ciudad de San José de Cúcuta y su área metropolitana", Plan de estudios de Ingeniería de Producción Industrial. San José de Cúcuta. Universidad Francisco de Paula Santander. Facultad de Ingeniería, Año 2008.

[12]. SANCHEZ MOLINA, Jorge. Diagnóstico general del sector cerámico del área metropolitana de Cúcuta. Grupo de Investigación en Tecnología Cerámica GITEC. San José de Cúcuta. Universidad Francisco de Paula Santander. Facultad de ciencias Básicas. Año 2009.
[13]. SANCHEZMOLINA, Jorge. Estrategias para la competitividad del cluster de la cerámica del Área Metropolitana de Cúcuta. San Cristóbal, Venezuela, 2010. Universidad Nacional Experimental del Táchira. Decanato de posgrado, Maestría en Gerencia de Empresas, Mención Industria

[14]. WEIERS, Ronald. Investigación de Mercados, editorial Prentice Hall, 1986.

[15]. INSTITUTO GEOLOGICO Y MINERO DE ESPAÑA. Arcilla, En línea, consultado el 25 de julio de 2011 en <http://www.igme.es/ internet/PanoramaMinero/actual/ ARCILLAS09.pdf>

[16]. ASOCIACIÓN ESPAÑOLA DE FABRICANTES DE LADRILLOS Y TEJAS DE ARCILLA COCIDA. Datos del Sector 2009. En Línea disponible en <http://www.hispalyt.es/estadistica. asp?id_rep=3315>

[17]. QUIBIT CLUSTER TECHNOLOGY BASED CONSORTIUM. Estrategia para la conformación de Cluster y plan estratégico para la cadena de cerámica en Cúcuta. Segundo informe. Bogotá D.C. 8 de noviembre de 2007.

[18]. TORRES, David y SANCHEZ, Johan. Análisis del impacto socioeconómico del sector cerámico del Área Metropolitana de Cúcuta a raíz de la problemática actual de las relaciones bilaterales entre Colombia y Venezuela. Cúcuta, 2011. Trabajo de grado Ingeniero de producción industrial. Universidad Francisco de Paula Santander, Facultad de ingenierías.

[19]. PUBLICA SOCIEDAD LIMITADA. Segmentación de los hornos empleados para fabricación de cerámica roja. Revista técnica cerámica No. 359, ISSN 0211-7290, 2007. 